\title{
SEMI-AUTOMATIC CLASSIFICATION OF LESION PATTERNS IN PATIENTS WITH CLINICALLY ISOLATED SYNDROME
}

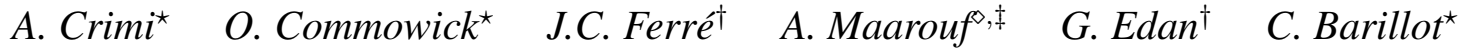 \\ * INRIA $\quad{ }^{\dagger}$ CHU Rennes $\quad{ }^{\circ}$ CRMBM-CNRS-Aix-Marseille University $\quad{ }^{\ddagger}$ CHU Reims
}

\begin{abstract}
Multiple sclerosis (MS) is neuro-degenerative disease of the Central Nervous System characterized by the loss of myelin. A Clinically Isolated Syndrome (CIS) is a first neurological episode caused by inflammation/demyelination in the central nervous system which may lead to MS. Better understanding of the disease at its onset will lead to a better discovery of pathogenic mechanisms, allowing suitable therapies at an early stage. We propose an automatic segmentation algorithm for two different contrast agents, used within a framework for early characterization of CIS patients according to lesion patterns, and more specifically according to the nature of the inflammatory patterns of these lesions. We expect that the proposed framework can infer new prospective figures from the earliest imaging signs of MS since it can provide a classification of different types of lesions across patients. The lesion detection algorithm based on intensity normalization and subtraction of the used MRI data is a pivotal step, since it avoids the time-demanding task of manual delineation.
\end{abstract}

Index Terms - Segmentation, MS, USPIO, longitudinal Study

\section{INTRODUCTION}

Multiple Sclerosis (MS) is an acquired inflammatory, demyelinating disease which causes disabilities in young adults and it is very common in the northern hemisphere. The natural evolution of MS is highly variable and it is still not fully understood, though its nature seems related to a two-phase autoimmune response[1]. At the moment it is impossible to predict accurately the severity, nature and progression for each patient. The MS onset can be studied on CIS patients, which are patients who experienced a first attack suggestive of demyelination, but do not fulfill yet the criteria for multiple sclerosis even if they are very likely to develop it [1]. Previous longitudinal studies show some correlation between the information gathered by MRI volumes and disease development. However, some results are controversial. There is a general agreement that the total lesion load (TLL) evaluated on T2 images correlates with future disabilities, though there is no clear consensus [1]. In acute MS, macrophages act more strongly than T-cells [2]. Hence, studying the early deposits of macrophages using specific markers is expected to lead to a better understanding of the pathology and of its evolution. This can be done monitoring patients at the onset of the disease and using a novel contrast agent, called Ultrasmall Superparamagnetic Iron Oxide (USPIO) sensitive to macrophages activity. USPIO can show different enhancements compared to the traditional Gadolinium (Gd) due to its nature of highlighting macrophages [3, 4]. The use of USPIO on CIS patients has been previously investigated using manually delineated lesions [5], this study is here extended comparing manual delineated lesions with automatically detected. The described framework might lead to a better understanding of the early pathogenic mechanisms in MS, and allow suitable therapeutic strategies at an early stage. The validation of these clusters is given by a statistical analysis, by a cross-validation and by a correlation with future TLL (enhanced and unenhanced) by 15 months follow up. with long term disabilities [1], though the aim is not to propose a biomarker to predict future TLL. A previous investigation has been carried out using manual delineation [5], however for the sake of reproducibility the framework has been extended with an automatic lesion detection. Manual delineation is a cumbersome and time-demanding task which with the proposed algorithm can be reduced. Moreover, the focus of this paper is on comparing the results obtained using the automatic and manual segmentation.

\section{MATERIALS AND METHODS}

The cohort comprises 25 CIS patients (17 women and 8 men) from different centers and which were assessed RRMS by follow-up. The MRI volumes were acquired by different hospitals using a 3T scanner according to a multi-center protocol. For each patient, at $m$ time points (two), we acquired different MRI volumes with an interval of three months, and we call these acquisitions $m_{0}$ and $m_{3}$, where the numeric index represents the month of the acquisition. The acquisitions of volumes were limited to these two time-points, because the $90 \%$ of the patients after $m_{3}$ were diagnosed with clinical MS and start undergoing treatments based on interferon which could interfere with the natural course of the disease. The acquired volumes for these time points were: a T1 volume without contrast agent, T1 Gdenhanced, and a T1 USPIO-enhanced. After 15 months after $m_{3}$, a $\mathrm{T} 2$ volume was acquired only for follow-up validation, this is considered as a further time point $m_{18}$. Seven patients did not present active lesions at the first two time points, but they will be defined as MS patients as well later. These patients have been excluded from the clustering described in the following sections, but their evolution in terms of future TLLs is also considered. All the T1 volumes have a voxels with voxelsize $1 \times 1 \times 3 \mathrm{~mm}^{3}$ (TE= $8.5 \mathrm{~ms}$, TR=500ms), while the T2 volumes have a voxels with voxelsize $1 \times 1 \times 1 \mathrm{~mm}^{3}$ $(\mathrm{TE}=88 \mathrm{~ms}, \mathrm{TR}=6.5 \mathrm{~ms})$. The USPIO-enhanced MRI were acquired after 1 day delay to let the macrophages go in the inflammatory area, though the intravenous injection of USPIO was performed on the same day of the Gd-enhanced acquisition. Gd and USPIO do not highlight the same phenomenon: Gd shows the blood brain barrier breakdown whereas USPIO highlight the inflammatory process related to macrophages. The follow-up TLLs are obtained from T2 volumes 15 months after the last baseline time point, namely at $m_{18}$. A radiologist performed the manual delineation of the enhanced lesions on $\mathrm{T} 1$ and of all the lesions on $\mathrm{T} 2$ volumes. T2 lesions are here used only as a further validation, their automatic delineation is beyond the purpose of this paper and has been already studied [1]. 


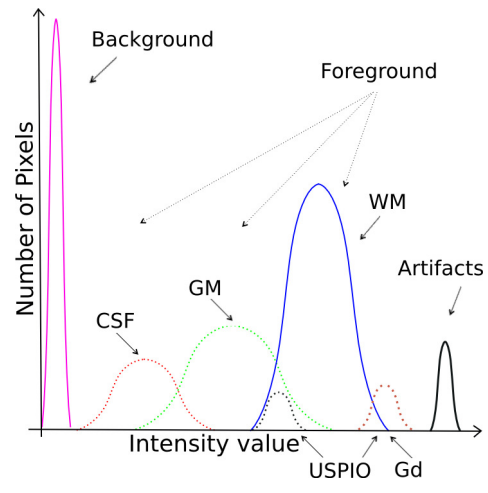

Fig. 1. Intensity histogram example of a Brain T1- MRI and its main modes representing a specific anatomical tissue, such as cerebrospinal fluid (CSF), gray matter (GM), white matter (WM), and MS lesions. The alignment is performed only considering the WM peak, the other Gaussians despite existing are aligned considering them as tails of the WM Gaussian.

\subsection{Semi-Automatic features extraction}

The focus of the paper is to discover lesion patterns that can be useful to identify MS patients which can undergo severe course of the disease. Some algorithms have been proposed for segmenting contrast enhanced lesions [6, 7]. However, since the lesions highlighted with USPIO have a very mild intensity they can be cumbersome for the common algorithms of lesions segmentation. Therefore, an algorithm based on subtraction between pre-contrast volume and postcontrast volume has been devised. Before the subtraction, some preprocessing is required such as noise reduction [8] and inhomogeneity correction [9]. Despite the positive effects of these processing steps, direct comparison of MRI volumes is not yet possible due to further variations related to different scanners or settings, but also to different stages of a disease even for the same patient. Some techniques have been proposed to standardize the intensity of MRI volumes starting from a template, a comparison of these techniques is reported in [10]. The landmark-based method of Nyul et al. [11] matches landmarks on different histograms. This method and its evolutions $[12,13]$ have several advantages such as a small computational time without losing accuracy especially for MS lesions detection. This method assumes that the histogram of an MRI volume $\boldsymbol{I}_{\boldsymbol{a}}$ has three main modes: the background, the foreground and artifacts (the foreground it can be decomposed in further modes but it is here considered as a unique mode). Once the background and artifacts modes are neglected, the foreground mode can be easily aligned, by stretching the histogram according to the foreground mode and variance of a reference volume histogram $\boldsymbol{I}_{\boldsymbol{b}}$. All the foreground voxels $j$ of $\boldsymbol{I}_{\boldsymbol{a}}$ are therefore standardized using the following expression:

$$
f(j)=\frac{\sigma_{b}}{\sigma_{a}}\left(j-\mu_{a}\right)+\mu_{b},
$$

where $\sigma_{a}, \sigma_{b}, \mu_{a}$ and $\mu_{b}$ are the standard deviations and the means of respectively the foreground modes of $\boldsymbol{I}_{\boldsymbol{a}}$ and $\boldsymbol{I}_{\boldsymbol{b}}$. In particular the alignment is performed considering the histograms using as mean value the peak representing the average intensity value for the WM and the variance given by all the values ( Figure 1 depicts the histogram showing all the main modes, despite the reference mode is mainly WM). In this paper the focus is on detecting active lesions while keeping the number of False Positives (FP) low rather than a precise delineation of lesion boundaries. The intensity normalization followed by volume subtraction leads to an acceptable segmentation

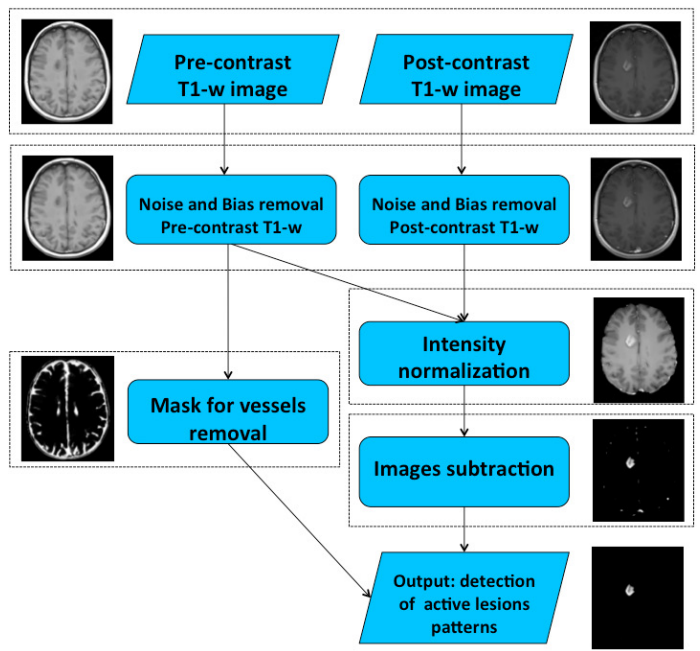

Fig. 2. Process workflow for extracting the features.

of the lesions. However, the process still highlights blood vessels carrying the contrast agent, the lesion extraction must be therefore refined suppressing the blood vessels. Some solutions have been proposed based on a special pulse sequence [6] or a prior segmentation of lesions on a registered T2 volume [14]. Relying on a prior T2 segmentation is not desirable since it is a challenging task itself and since it will require a good correspondence between the $\mathrm{T} 2$ lesions and the enhanced lesions. An alternative approach to vessel suppression was based on analyzing the curvature of the lesions within a Conditional Random Fields approach [7]. Despite this approach will reduce the number of necessary volumes and simplify the framework, it could not suppress small vessels with non-tubular appearance. Inspired by [6], a simpler but still reliable solution has been adopted. It was noticed that the vessels can be removed performing several post-processings. First all the voxels which are in a distance of ten voxels from the edge of the brain are removed. Then, using a Cerebro Spinal Fluid (CSF) mask, the voxels representing CSF are also removed. This mask is obtained from the probabilistic segmentation, thresholded with a very small threshold value th according to the intensity voxel (e.g. $t h=10$, using volumes with normalized intensity within the range $[0,255])$. In this study, this parameter has not been optimized or changed according to specific volumes, but it is supposed to be used equally for all the volumes. Despite the improvements introduced by the postprocessings, the automatic lesion segmentation is however not directly usable for the classification, since it can still highlight some vessels as FP, and hence it requires human intervention before the classification. Some lesions were detected as made of 2 independent lesions, which could cause improper classification, therefore morphological operators or human intervention is required. For these reasons the method is considered semi-automatic. Figure 2 depicts the segmentation workflow.

\subsection{Features representation}

Once the enhanced lesions are manually delineated or segmented for all $m$ time points (two in our case), they are aligned according to their position respectively for each time point. As in Crimi et al. [5], a tensor-like representation combined with a hollowness index was used. Practically, the lesions are represented as 3D spatial tensors and by an hollowness index $h_{i}$. To uniform the representation, the ring-like shapes have been considered as filled and the hollowness is measured and concatenated to this information. The hollowness 
is given by the ratio $h_{i}=\frac{A_{d_{i}}}{A_{f_{i}}}$, where $A_{d}$ is the lesion area directly obtained by all the segmentations, and $A_{f}$ is the same area with filled holes, this ratio should be 1 if there is no hole and becoming smaller than 1 if a hole is present. The ordered eigenvalues of these 3D tensors give a rotationally invariant feature representation: For each lesion at each time point, the coordinates of the $n$ voxels belonging to a specific lesion are collected in the data matrix $\boldsymbol{X}=\left[\left(x_{1}-\mu_{x}\right),\left(y_{1}-\mu_{y}\right),\left(z_{1}-\mu_{z}\right) \mid\left(x_{2}-\mu_{x}\right),\left(y_{2}-\mu_{y}\right),\left(z_{2}-\right.\right.$ $\left.\left.\mu_{z}\right)|\ldots|\left(x_{n}-\mu_{x}\right),\left(y_{n}-\mu_{y}\right),\left(z_{n}-\mu_{z}\right)\right]$ regardless of the intensity of the voxels. Here, $\mu_{x}, \mu_{y}$ and $\mu_{z}$ are the mean of the coordinates for the $n$ voxels. These values are used for defining a covariance matrix $\boldsymbol{\Sigma}$. All the three eigenvalues $\lambda$ obtained by the eigendecomposition of $\boldsymbol{\Sigma}$ and the hollowness index for all $m$ time points are the features representing the temporal evolution of the lesions. This means that for each single lesion, there is a feature vector $\boldsymbol{f}_{\boldsymbol{G d}}$ and

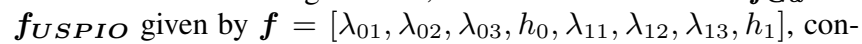
taining the values respectively for the MRI volume Gd-enhanced and USPIO-enhanced, the eigenvalues $\lambda$ are collected in decreasing order for each time point to be orientation independent. Once the lesions are represented for both contrast agent, the vectors $\boldsymbol{f}_{\boldsymbol{G d}}$ and $\boldsymbol{f}_{U S P I O}$ of each lesion are concatenated in a vector $\boldsymbol{g}_{G d-U S P I O}$. If one lesion has only either $\boldsymbol{f}_{G \boldsymbol{d}}$ or $\boldsymbol{f}_{U S P I O}$, the absence of the feature is represented by zeros. The same lesion present in different time points $m$, is aligned to the other time representation of itself. This is performed registering all the manual delineation volume of different time points for the same patient. If there is an overlap between the lesion of the different time point, then they are assessed automatically to be the same lesion and collected into the same $\boldsymbol{g}$ vector. The patient feature vector is instead given by all the cardinalities of the lesion pattern clusters $n_{C_{i}}$ present in a patient as $\boldsymbol{q}=\left[n_{C_{1}}, n_{C_{2}}, n_{C_{3}}\right], n_{C_{i}}=0$ if it is not present in a specific patient, this vector could be larger in case of more detected clusters. The following subsection describes how the lesion and patient clusters are obtained.

\subsection{Lesion evolutions and patient classification}

The framework is based on a two-layer classification. Initially lesion patterns are identified, then the discovered patterns are used to identify patients with high probability of future high TLL. These two steps are performed by an unsupervised clustering algorithm and a regression using the output of the first layer as the input of the second. The lesion classification is based on spectral clustering [15] since this methodology can handle complicated structure, or when the clusters have different sample sizes, which are circumstances likely to occur in our case. However, even with spectral clustering the first hurdle is the choice of the cluster number which can be estimated using a validity index. Many validity indices have been proposed, in this study a variation of the $\mathrm{Ng}$-Jordan-Weiss algorithm [15] is used. This method is strictly related to spectral clustering, where the number of clusters is discovered by analyzing the eigenvalues of the affinity matrix which represents the pairwise distances among all the samples. In particular, the number of clusters can be given by the number of eigenvalues $\lambda_{i}$ of the affinity matrix equaling 1. A robust version of the approach was proposed for the case of not well separated data [15], this version estimates all the necessary parameters, and performs the spectral clustering and k-means. Once the clustering of the lesions pattern is constructed, the detected $q$ vectors representing the number of each detected clusters can be used to identify patients with high probability of future high TLL. Although the aim is not to predict TLL, this correlation is given to relate the patient classification to their severity of the disease. This is performed using the regression model

$$
T L L=\beta_{1}\left(n_{C_{1}}\right)+\beta_{2}\left(n_{C_{2}}\right)+\beta_{3}\left(n_{C_{3}}\right),
$$

where the coefficients can be more in case of more detected clusters. The model examines the influence of the detected number of lesion patterns, since it is believed that some lesion patterns are more indicative of the severity of the disease. The parameters $\beta_{i}$ were estimated minimizing the Least-Squares linear regression given by $\sum\|\boldsymbol{Q} \boldsymbol{B}-\boldsymbol{T}\|^{2}$, where $\boldsymbol{B}$ is the vector containing all the $\beta_{i}, \boldsymbol{Q}$ is the matrix containing all the number of patterns per patient $q$ and $\boldsymbol{T}$ is vector containing the TLLs.

\section{RESULTS}

Comparing the enhancement for both contrast agents for the same lesions, it is noted that the USPIO-enhanced lesions show a different behavior than the Gd-lesions and are often visible as a mild ringing around the Gd-lesions as depicted in Figure 3. Figure 4 depicts

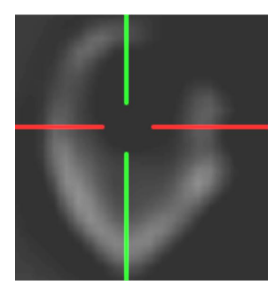

(a)

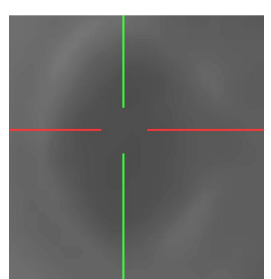

(b)

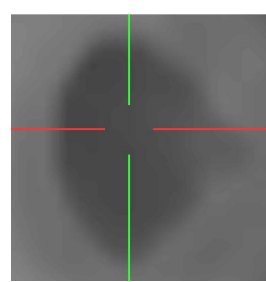

(c)
Fig. 3. The same lesion at the same time point: (a) Gd, (b) USPIO and (c) pre-contrast.

a slice of the resulting volume of respectively the subtraction between the MRI without contrast agent, MRI Gd-enhanced and with USPIO-enhanced. The lesions manually detected by the radiologist were 103 Gd-lesions and 24 USPIO-lesions on 100 volumes (4 for each patients). Comparing the automatic to the manual segmentations only $2 \mathrm{FN}$ were found, and despite the post-processing $489 \mathrm{FP}$ were detected $(4.89 \pm 5.19 \mathrm{FP}$ as a mean and standard deviation per volume). The FP removal was performed comparing the manual delineation, in clinical practice this can be done asking a further validation by a radiologist, which is an acceptable nuisance compared to the cumbersome process of manual delineation. A dice index is not reported since the aim is on detecting active lesions while keeping the number of FN low rather than a precise delineation of lesion boundaries. The patients of a center presented less lesions and also less FP. The statistical significance of the detected patterns [5] was assessed by computing the cluster separation of the lesion patterns, which is defined as the average distance between each cluster centroids and the other centroids. This analysis repeated over 1000000 resampled permutations led to $\mathrm{p}$-values $<0.0001$. The TLL and number of detected patterns are used to build the regression model of equation (2), which seems to behave well since the mean obtained $R^{2}$ coefficient performed in a cross-validation manner was 0.65 for the automatically detected lesions against 0.68 for the manually delineated. The validity index used on the manually delineated lesions detected three clusters, a non specific cluster $C_{1}$ comprising mainly only Gd-enhanced lesions, and other two clusters $C_{2}$ and $C_{3}$, representing relatively average and big lesion with both Gd and USPIO, these latter two clusters were also correlating with high TLL, a more complete description is given in [5]. The same validity index 

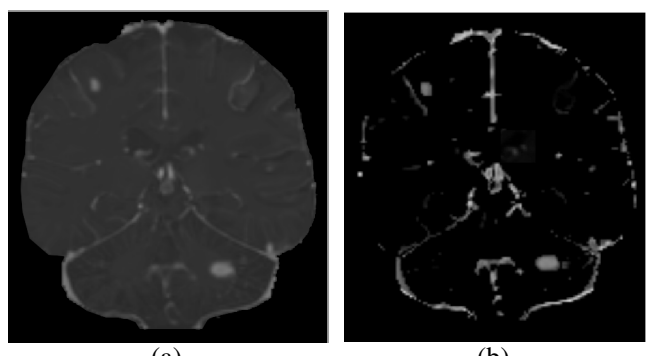

(a)

(b)

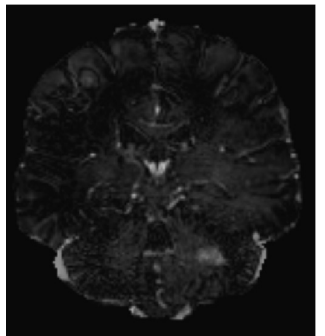

(c)

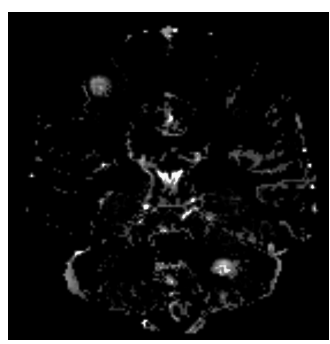

(d)

Fig. 4. The same difference slice between MRI and Gd-enhanced MRI respectively without (a), with histogram standardization(b), USPIO-enhanced MRI respectively without (c) and with histogram standardization (d). In these images the vessels removal step has not been processed

used on the automatically obtained features gave similar results, but 5 clusters were identified. An identical cluster $C_{1}$ with non specific Gd-enhanced lesions, and similarly to the manually delineated lesions big lesions presenting both Gd and USPIO were distributed in the other clusters but in 4 different clusters rather than 2. Although these lesion patterns appear different compared to the lesion patterns comprised in the generic cluster.

\section{DISCUSSION}

The proposed framework can classify in a quasi-automatic way lesions and MS patients from the very early stages ( first 2 scans), this classification correlates to the TLL by 15 months follow-up after the second scan. This framework comprises an ad hoc segmentation based on subtraction of volume and intensity normalization. Using more advanced methodology for intensity normalization [12, 13], more precise lesion segmentations were obtained but more FP derived by partial volume effects were also generated, making the improvements counter-productive. The FP reported in section 3 are present either due to imprecise intensity normalization or imprecise CSF segmentation, parameters optimization could improve the results. Similarly to [5], the clustering algorithm allowed the definition of patterns according to difference in size and the presence of both contrast agents. It can be hypothesized that the disease severity is related to the presence of some of these patterns. This suggests that a risk assessment should be performed using both contrast agents. The different behavior of USPIO, compared to Gd, leads to the hypothesis that USPIO can help to mark lesions with high risk to evolve to a more severe course, though has been suggested that it could also highlight healing process [4]. Although promising, the predictive value of USPIO needs to be validated on larger populations. The tensor-like representation for lesions is motivated by the rotationally invariant eigenvalues and the analogy to PCA. However, to complement the tensor-like representation a clear indication of the

hollowness of the lesion has been added in such a way to discriminate focal from ringing-like lesions. Future works are given by the inclusion of other MRI measurements (such as Magnetization Transfer or Diffusion MRI).

\section{CONCLUSION}

A novel paradigm for spatio-temporal analysis of MS lesions including an ad hoc segmentation has been described. It has been applied to a novel contrast agent (USPIO) that appears to give complementary information compared to the traditional Gd. An intensity normalization based algorithm is able to roughly segment the active lesions necessary for the classification. This patient classification performed at the very early stages- correlates with the future TLL after 15 months from the last used time point, this might indicate an even further evolution [1]. This finding could lead to more precise prognosis and more suitable treatments.

\section{REFERENCES}

[1] M. Filippi and et al., "Quantitative brain lesion load predicts the course of CIS," Neurology, vol. 44, pp. 635-41, 1994.

[2] A. Bitsch and et al., "Acute axonal injury in multiple sclerosis," Brain, vol. 123, pp. 1174-83, 2000.

[3] V. Dousset, "Correlation between clinical status and macrophage activity imaging in the central nervous system of rats," Ac. Radiology, vol. 9, pp. 156-9, 2002.

[4] M. Vellinga and et al., "Pluriformity of inflammation in MS shown by ultra-small iron oxide particle enhancement," Brain, vol. 131, pp. 800-07, 2008.

[5] A. Crimi and et al., "Multiple sclerosis lesions evolution in patients with CIS," in SPIE Medical Imaging, 2013.

[6] B. Bedell and P. Narayana, "Automatic segmentation of gadolinium-enhanced multiple sclerosis lesions," Magnetic Resonance Medicine, vol. 39, pp. 935-40, 1998.

[7] Z. Karimaghaloo and et al., "Automatic detection of Gdenhancing multiple sclerosis lesions in brain MRI using CRF," IEEE TMI, vol. 31, pp. 1181-94, 2012.

[8] P. Coupe and et al., "An optimized blockwise nonlocal means denoising filter for 3D magnetic resonance images," IEEE TMI, vol. 27, pp. 425-41, 2008.

[9] J.F. Mangin, "Entropy minimization for automatic correction of intensity nonuniformity," in IEEE Workshop on MMBIA, 2000, pp. 162-69.

[10] J.P. Bergeest and F. Jaeger, "A comparison of five methods for signal intensity standardization in MRI," in Bildverarbeitung fuer die Medizin, 2008, pp. 36-40.

[11] L. Nyul and et al., "New variants of a method of MRI scale standardization," IEEE TMI, vol. 19, pp. 143-50, 2000.

[12] P. Hellier, "Consistent intensity correction of MRI," in Proc. ICIP, 2003, pp. 1109-12.

[13] V. Noblet, Recalage non rigide d'images cerebrales $3 D$ avec contrainte de conservation de la topologie, Ph.D Thesis, 2006.

[14] S. Datta and et al., "Segmentation of gadolinium-enhanced lesions on mri in multiple sclerosis," J. Magn. Reson. Imaging, vol. 25, pp. 932-37, 2007.

[15] L. Zelnik-Manor and P. Perona, "Self-tuning spectral clustering," in NIPS, 2004, vol. 17. 